\title{
Seguridad hídrica: Gestión del agua en comunidades rurales del Pacífico Norte de Costa Rica
}

\author{
Water Security: Water Management in Rural Communities of the Northern Pacific \\ Region of Costa Rica
}

\section{Andrea Suárez Serrano ${ }^{a}$, Álvaro Baldioceda Garro ${ }^{b}$, Guillermo Durán Sanabria $^{c}$, Johanna Rojas Conejo ${ }^{d}$, Daniela Rojas Cantillano ${ }^{e}$, Anny Guillén Watson ${ }^{f}$.}

[Recibido: 18 de setiembre 2018, Aceptado: 4 de marzo 2019, Corregido: 29 de marzo 2019, Publicado: 1 de julio 2019]

\section{Resumen}

Se seleccionó un grupo de 9 Asociaciones Administradoras de Acueductos Rurales (ASADAS) ubicadas en el Pacífico Norte de Costa Rica, con el propósito de evaluar su gestión y conocer las estrategias implementadas durante el periodo de sequía (2014-2016). Se valoraron 5 componentes básicos de gestión: administrativa y financiera, comercial, comunal, recurso hídrico y gestión de los sistemas de agua, con el fin de determinar si son adecuadas para proveer agua segura a las comunidades. Además, se analizó la calidad del agua potable de cada ASADA, aplicando el nivel 1, según el Reglamento para la Calidad del Agua Potable (38924-S). Una de las ASADAS estudiadas presentó desarrollo alto en su nivel de gestión; 3, un desarrollo bajo, y 5 una gestión débil. En 2 ASADAS, se detectó la presencia de coliformes fecales y E. coli durante la época lluviosa. Solamente 3 de las 9 ASADAS estudiadas cumplieron con los rangos óptimos de cloro libre residual, lo cual compromete la calidad del agua con la que se abastece a la población. Se concluye que la fragilidad de las ASADAS evaluadas ante un evento hidrometeorológico es alta, debido a la antigüedad de los sistemas, falta de mantenimiento y poca capacidad de sus tanques, lo que incrementa el riesgo durante eventos extremos (sequías e inundaciones). Además, las ASADAS exploradas mostraron, principalmente, una gestión baja o débil; por consiguiente, el agua que se distribuye a las comunidades podría afectar la seguridad hídrica de aquellas abastecidas, debido a que no siempre está disponible cuando se necesita y no cumple con algunos de los parámetros básicos de potabilidad.

Palabras claves: calidad y cantidad del agua; gestión comunitaria del agua; suministro de agua potable.

\section{Abstract}

A group of 9 Associations for the Administration of Rural Aqueducts (ASADAS) located in the Northern Pacific region of Costa Rica were selected to evaluate their management and document the strategies they implemented during the drought of 2014-2016. Five basic management components were evaluated: administrative and financial, commercial, communal, water resources, and management of water systems, to determine if management was

a Coordinadora general. Centro de Recursos Hídricos para Centroamérica y el Caribe (HIDROCEC-UNA), Universidad Nacional, Costa Rica. andrea.suarez.serrano@una.ac.cr, https://orcid.org/0000-0002-1930-3381

b Investigador en el área de tratamiento y reutilización de agua. Centro de Recursos Hídricos para Centroamérica y el Caribe (HIDROCECUNA), Universidad Nacional, Costa Rica. alvaro.baldioceda.garro@una.ac.cr, https://orcid.org/0000-0003-1121-463X.

c Investigador en el área de gestión de cuencas hidrográficas. Centro de Recursos Hídricos para Centroamérica y el Caribe (HIDROCEC-UNA). Universidad Nacional, Costa Rica. guillermo.duran.sanabria@una.ac.cr, https://orcid.org/0000-0002-1944-9524

d Investigadora en el Laboratorio de Análisis Fisicoquímicos. Centro de Recursos Hídricos para Centroamérica y el Caribe (HIDROCEC-UNA). Universidad Nacional, Costa Rica. johanna.rojas.conejo@una.ac.cr, https://orcid.org/0000-0002-9001-3694

e Investigadora en el Laboratorio de Análisis Fisicoquímicos. Centro de Recursos Hídricos para Centroamérica y el Caribe (HIDROCEC-UNA). Universidad Nacional, Costa Rica. daniela.rojas.cantillano@una.ac.cr, https://orcid.org/0000-0002-1977-3738

f Investigadora en Laboratorio de Microbiología Ambiental. Centro de Recursos Hídricos para Centroamérica y el Caribe (HIDROCEC-UNA). Universidad Nacional, Costa Rica. anny.guillen.watson@una.ac.cr, https://orcid.org/0000-0001-6719-1276

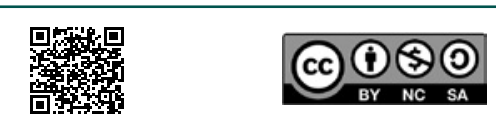

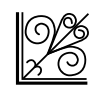

euna 


\section{Revista de CIENCIAS AMBIENTALES Tropical Journal of Environmental Sciences}

Revista de Ciencias Ambientales (Trop J Environ Sci)

e-ISSN: 2215-3896

(Julio-Diciembre, 2019) . Vol 53(2): 25-46

DOI: https://doi.org/10.15359/rca.53-2.2

Open Access: www.revistas.una.ac.cr/ambientales e-mail: revista.ambientales@una.ac.cr

Suárez A., Baldioceda A., Durán G., Rojas J., Rojas D., Guillén, A.

adequate to provide safe water to the communities. In addition, the quality of drinking water of each ASADA was analyzed, using Level 1 of the Regulation for Quality of Drinking Water (38924-S) as a criterion. One of the ASADAS studied had a high level of management capability; 3 had a low level of management capability, and 5 had weak management. The presence of fecal coliforms and E. coli was detected during the rainy season in 2 ASADAS. Only 3 of the 9 ASADAS analyzed met the optimal ranges for free residual chlorine, which endangers the quality of the water supplied to the population. It is concluded that the ASADAS evaluated are highly fragile when confronted by a hydrometeorological event, due to the age of systems, lack of maintenance, and the limited capacity of their storage tanks, which increases risk during extreme events (droughts and floods). In addition, the ASADAS evaluated had mostly low or weak management capabilities; consequently, the water that is distributed to communities could affect water security of the communities that they serve, since this water is not always available when needed and does not comply with some of the basic parameters of potability.

Keywords: community water management; drinking water supply; quality and quantity of water.

\section{Introducción}

El agua es fundamental para todos los sectores sociales, económicos, así como para la base de recursos naturales de la cual depende el mundo entero (UNESCO, 2017). En la Cumbre de Desarrollo Sostenible realizada en septiembre del 2015, 193 líderes del mundo se comprometieron con 17 objetivos, según la Organización de las Naciones Unidas, de los cuales, para efectos de este artículo, el más relevante es el 6: Garantizar la disponibilidad y la gestión sostenible del agua, así como el saneamiento para todos, servicios manejados de manera segura (metas 6.1 y 6.2 de los ODS).

El acceso a agua segura y a servicios de saneamiento sigue siendo excesivamente bajo en la mayoría de las áreas rurales. Costa Rica tiene uno de los índices de cobertura más altos (número de casas conectadas a tuberías de agua) para el agua potable en las zonas rurales de América Latina (OMS, 2013). La cobertura promedio del servicio de agua potable intradomiciliar es de 92 \%, en San José, 96.6 \%; en Guanacaste, 88.7 \%, y en Limón, 77.3 \% (INEC, 2015).

Esta alta cobertura se debe en un $98.2 \%$ a la labor del ente gubernamental, Instituto de Acueductos y Alcantarillados (AyA); en un $25.5 \%$ en el nivel nacional, al trabajo de las Asociaciones Administradoras de Acueductos Rurales (ASADAS), y, en un $25 \%$, a acueductos municipales (AyA, 2016). Sin embargo, solo se menciona la cobertura, no la calidad; en el 2010, de 1067 ASADAS evaluadas por el Laboratorio Nacional de Aguas, se encontró que solamente 602 ofrecen agua potable (56.4\%) y 465 agua no potable (43.6 \%). Lo anterior refleja que la calidad del agua se distribuye de manera desigual en Costa Rica; por ejemplo, en la capital, San José, el $93.2 \%$ de la población recibe agua de calidad potable, a diferencia de Alajuela, con solo el $71.1 \%$ y de Guanacaste, con el $74.8 \%$ (GWP, 2011).

La Organización para la Cooperación y el Desarrollo Económicos $(\mathrm{OECD}, 2013)$ indica que lograr seguridad hídrica significa mantener en niveles aceptables 4 tipos de riesgos asociados al agua: (1) riesgo de escasez (incluyendo sequías), (2) riesgo de calidad inadecuada (la falta de agua de calidad adecuada para un propósito o uso determinado), (3) riesgo de exceso (incluyendo

\begin{tabular}{|c|c|c|c|c|c|}
\hline 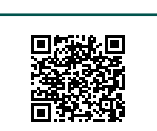 & (c) (1) (1) () () & 8 & $\frac{12 \% 6}{\frac{O O O}{20}}$ & 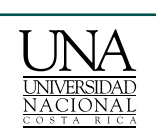 & 26 \\
\hline
\end{tabular}




\section{Revista de CIENCIAS AMBIENTALES Tropical Journal of Environmental Sciences}

Revista de Ciencias Ambientales (Trop J Environ Sci)

e-ISSN: 2215-3896

(Julio-Diciembre, 2019) . Vol 53(2): 25-46 DOI: https://doi.org/10.15359/rca.53-2.2

Open Access: www.revistas.una.ac.cr/ambientales e-mail: revista.ambientales@una.ac.cr Suárez A., Baldioceda A., Durán G., Rojas J., Rojas D., Guillén, A.

inundaciones) y (4) riesgo de afectar la resiliencia de los sistemas de agua dulce (se supera la capacidad de las aguas superficiales y subterráneas, así como sus interacciones, rebasando sus umbrales y causando daños irreversibles en las funciones tanto hidráulicas como biológicas del sistema). Todos estos riesgos se podrían incrementar en el contexto de cambio climático. El quinto informe del Grupo Intergubernamental de Expertos sobre el Cambio Climático (IPCC por sus siglas en inglés) advierte que los riesgos del cambio climático sobre los recursos hídricos aumentaron significativamente con el acrecentamiento de las emisiones de gases de efecto invernadero; que se reducirán las cantidades de agua renovable superficial y subterránea en zonas subtropicales secas, y que se espera un deterioro de la calidad del agua, entre otros efectos (IPCC, 2014).

Varios autores han conceptualizado el término de seguridad hídrica (De Stefano y Llamas, 2013; Grey y Sadoff, 2007; World Water Forum y Global Water Partnership, 2000). Una definición más reciente, que incorpora lo expuesto por Grey y Sadoff (2007), es escrita por Zeitoun (2011): el nivel de riesgos relacionados con el agua aceptable para los humanos y los ecosistemas, unido con la disponibilidad de agua en calidad y cantidad suficiente para apoyar medios de vida, seguridad nacional, salud humana y servicios ecosistémicos.

Existen pocos estudios que evalúen la gestión comunitaria de las organizaciones comunales de acueductos rurales. Las indagaciones existentes se han enfocado en la sostenibilidad de estas organizaciones de áreas rurales y en los factores que influyen en su desempeño, en el contexto de sequías estacionales (Madrigal-Ballestero y Naranjo, 2015; Navarro-Garro, Murillo, Segura y Ugalde, 2013).

Durante la sequía provocada por el fenómeno El Niño-Oscilación del Sur (ENOS) (20142016), en el Pacífico Norte, algunas comunidades abastecidas por ASADAS y otras autoabastecidas con pozos artesanales perdieron su fuente de agua. A partir de esta situación, las instituciones del Estado, entre estas MINAE, AyA, SENARA, Ministerio de Agricultura y Ganadería (MAG), Instituto de Desarrollo Rural (INDER), gobiernos locales, ASADAS y organizaciones privadas, etc., crearon el Plan Integral de Abastecimiento de Agua para Guanacaste (PIAAG), que incorpora diversos proyectos de todas las instituciones (Astorga, 2016). Además, se ha determinado que esta región presenta una insuficiente inversión en infraestructura hidráulica y una débil gestión del recurso hídrico (Zeledón, 2016).

En el Plan Nacional de Desarrollo 2015-2018, se describen varios proyectos hidráulicos de abastecimiento de agua en las comunidades, para el desarrollo de la provincia, así como acciones habilitadoras con miras a asegurar el aprovechamiento óptimo del recurso hídrico disponible, por ejemplo, el Proyecto Sistema de Abastecimiento de Agua para la Cuenca Media del Río Tempisque y Comunidades Costeras (Paacume) (Coto, 2016). Sin embargo, es importante tomar en cuenta que no se tiene una fecha clara para la realización y operación de estos proyectos, al igual que cuáles ASADAS serían beneficiadas directamente y de manera prioritaria.

Este estudio se realizó en el Pacífico Norte de Costa Rica, en la provincia de Guanacaste, que por su ubicación geográfica y sus condiciones socioeconómicas es, desde el punto de vista de la gestión integrada del recurso hídrico, la más vulnerable y amenazada por el cambio

\begin{tabular}{|c|c|c|c|c|c|}
\hline 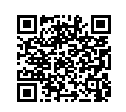 & (c) & 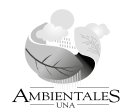 & $\frac{O \%}{20}$ & $\frac{\text { UNA }}{\frac{\text { UNNVERSIDAD }}{\frac{N A C I O N A L}{\text { NASTARACA }}}}$ & 27 \\
\hline
\end{tabular}




\section{Revista de CIENCIAS AMBIENTALES Tropical Journal of Environmental Sciences}

Revista de Ciencias Ambientales (Trop J Environ Sci)

e-ISSN: 2215-3896

(Julio-Diciembre, 2019) . Vol 53(2): 25-46 DOI: https://doi.org/10.15359/rca.53-2.2

Open Access: www.revistas.una.ac.cr/ambientales e-mail: revista.ambientales@una.ac.cr Suárez A., Baldioceda A., Durán G., Rojas J., Rojas D., Guillén, A.

climático. Ha sido afectada históricamente por grandes sequías y fenómenos extremos que impactan los recursos públicos, la base socioeconómica del país, y vulneran la gobernabilidad democrática de dicha provincia (MIDEPLAN, 2017). Esto podría incrementar el nivel de conflictividad por el uso del agua, observado en el sitio desde hace ya 20 años, principalmente, entre el sector turismo, la agricultura y las comunidades (Kuzdas y Wiek, 2014; Ramírez, 2007).

Las organizaciones comunitarias de agua potable como las ASADAS son las proveedoras más importantes de agua en áreas rurales y juegan un papel clave tanto en la descentralización como en los procesos de democratización costarricenses (Astorga, 2016). De aquí la importancia de fortalecer estas organizaciones comunales, por su papel fundamental en la dotación de agua para consumo. Por lo tanto, se seleccionó un grupo de 9 Asociaciones Administradoras de Acueductos Rurales (ASADAS), ubicadas en el Pacífico Norte de Costa Rica, con el objetivo de evaluar su gestión y conocer las estrategias implementadas durante el periodo de sequía (2014-2016), con el fin de determinar si la gestión comunitaria es adecuada para dar seguridad hídrica a las zonas rurales que abastecen.

\section{Metodología}

\section{1 Área de estudio}

La provincia de Guanacaste (Pacífico Norte de Costa Rica) posee una estacionalidad climática marcada (épocas seca y lluviosa); históricamente se han reportado importantes eventos de sequías provocados por el fenómeno El Niño-Oscilación del Sur (ENOS) (Sánchez-Murillo, Durán-Quesada, Birkel, Esquivel-Hernández y Boll, 2016). En general, las sequías en Costa Rica son recurrentes pero aperiódicas, en promedio se registra una condición seca extrema cada 8 años, que hace disminuir la precipitación anual en un $17 \%$ y afecta el $83 \%$ del área cubierta por estaciones meteorológicas (Retana, 2012). Sin embargo, la peor sequía ha sido la vivida en el periodo 2014-2016, catalogada como análoga en su comportamiento a la de 1997 1998, acorde con el Programa sectorial de cambio climático y gestión del riesgo a desastres de la Secretaría Ejecutiva de Planificación Sectorial Agropecuaria del Ministerio de Agricultura y Ganadería (MAG). En el 2014, el Gobierno de Costa Rica decretó el estado de emergencia nacional (Presidencia de la República, 2014), debido a la situación provocada por la influencia del fenómeno El Niño, específicamente en 11 cantones de Guanacaste y 8 del Pacífico Central, a través del decreto 38642 MP-MAG.

\subsection{Selección de los casos de estudio}

En Guanacaste, existen 317 Asociaciones Administradoras de Acueductos Comunales (ASADAS), además de Comités de Agua (CAARS) y asociaciones de usuarios. Del total de ASADAS, únicamente 186 poseen convenio de delegación con el AyA, 175 tienen menos de 200 abonados, 60 no cuentan con micromedición y un número mayor no determinado no trabaja

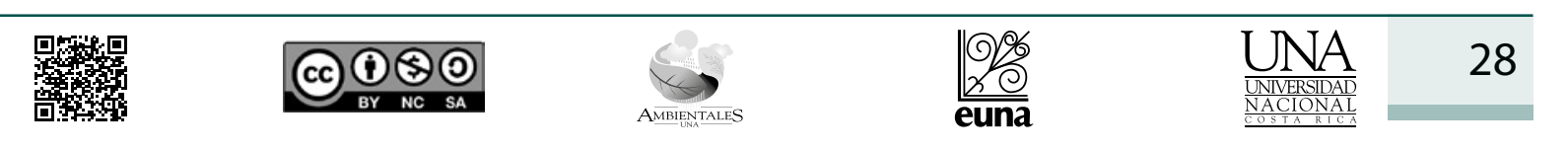




\section{Revista de CIENCIAS AMBIENTALES Tropical Journal of Environmental Sciences}

Revista de Ciencias Ambientales (Trop J Environ Sci) e-ISSN: 2215-3896

(Julio-Diciembre, 2019) . Vol 53(2): 25-46 DOI: https://doi.org/10.15359/rca.53-2.2 Open Access: www.revistas.una.ac.cr/ambientales e-mail: revista.ambientales@una.ac.cr Suárez A., Baldioceda A., Durán G., Rojas J., Rojas D., Guillén, A.

con macromedición. Nicoya posee el mayor número de ASADAS (alrededor de 80) y además, de los 11 cantones de Guanacaste (Alfaro, 2018), el que tiene la mayoría de ASADAS con menos de 100 abonados. Tomando en cuenta lo anterior, se eligió como estudio de caso a un grupo de 9 ASADAS de dicho cantón, a las que se les denominó alfabéticamente de la A a la I (Figura 1).
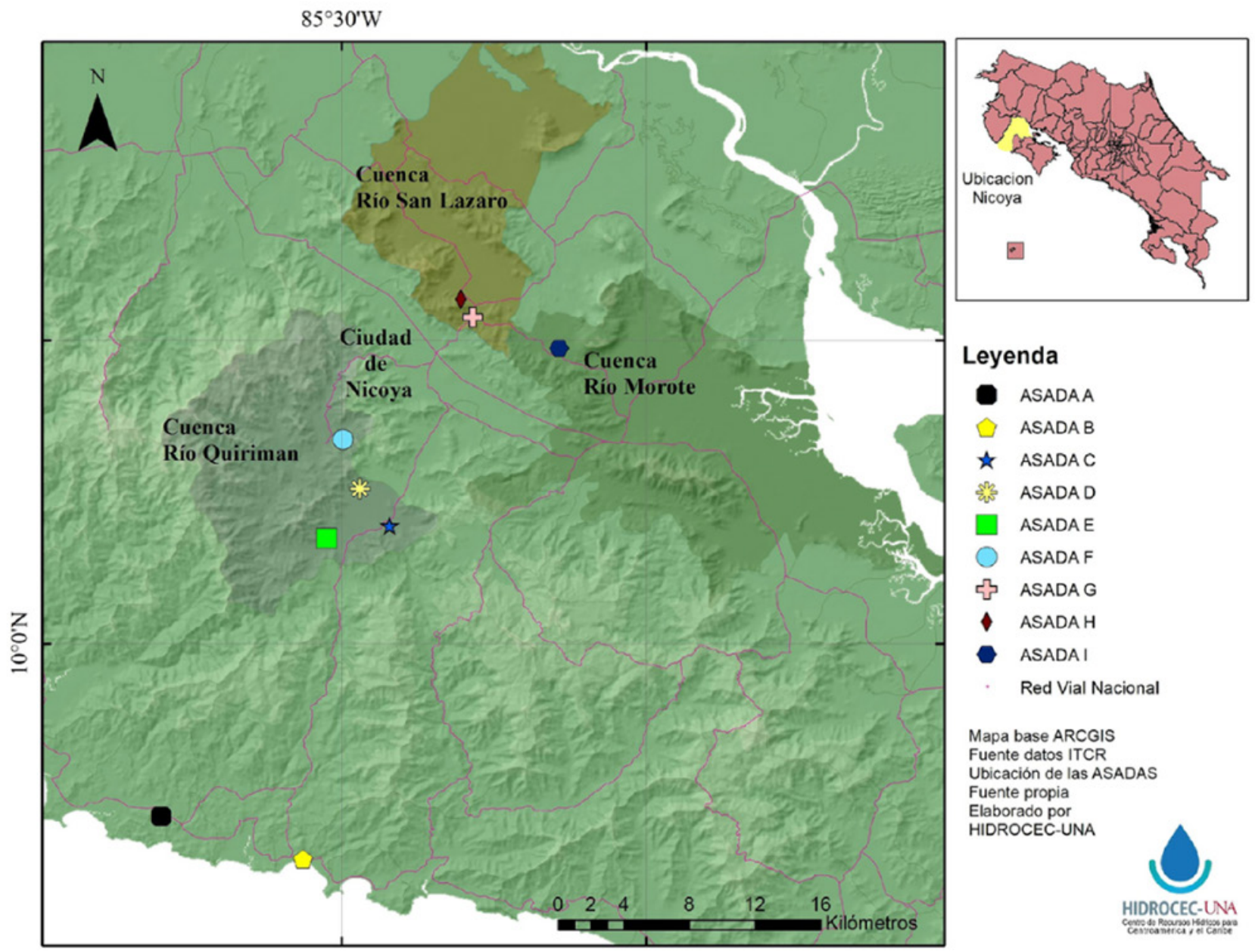

Figura 1. Área en estudio, ubicación de las ASADAS estudiadas y sus principales cuencas en el Pacífico Norte de Costa Rica (2016-2017).

En el Cuadro 1, se muestra una caracterización general de la selección. La ASADA B cuenta con la mayor población abastecida ( 3000 personas), mientras que la ASADA F, la menor (40 personas) y el resto se ubica en un rango de entre 400 y 1500 personas. Con respecto a la longitud de la tubería de los sistemas de agua potable, el rango está entre los 2 y los $11 \mathrm{~km}$. La

\begin{tabular}{|c|c|c|}
\hline 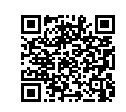 & (c) (i) () () & 29 \\
\hline
\end{tabular}




\section{Revista de CIENCIAS AMBIENTALES Tropical Journal of Environmental Sciences}

Revista de Ciencias Ambientales (Trop J Environ Sci)

e-ISSN: $2215-3896$

(Julio-Diciembre, 2019) . Vol 53(2): 25-46

DOI: https://doi.org/10.15359/rca.53-2.2

Open Access: www.revistas.una.ac.cr/ambientales e-mail: revista.ambientales@una.ac.cr Suárez A., Baldioceda A., Durán G., Rojas J., Rojas D., Guillén, A.

antigüedad de los acueductos osciló de 20 a 38 años, la ASADA B es el acueducto más antiguo, la profundidad de los pozos se encuentra entre 30 y $80 \mathrm{~m}$, así como el caudal, entre 1.5 y $10 \mathrm{l} / \mathrm{s}$.

Cuadro 1. Caracterización general de las ASADAS evaluadas (longitud total del sistema, población abastecida, antigüedad del acueducto, profundidad del pozo y caudal)

\begin{tabular}{cccccc}
\hline ASADA & $\begin{array}{c}\text { Longitud total de } \\
\text { la tubería del sistema }(\mathbf{k m})\end{array}$ & $\begin{array}{c}\text { Población } \\
\text { abastecida } \\
\text { (personas) }\end{array}$ & $\begin{array}{c}\text { Antigüedad del } \\
\text { acueducto } \\
(\mathbf{a n ̃ o s})\end{array}$ & $\begin{array}{c}\text { Máxima profundidad } \\
\text { Pozo }(\mathbf{m})\end{array}$ & $\begin{array}{c}\text { Caudal } \\
(\mathbf{l} / \mathbf{s})\end{array}$ \\
\hline A & 9 & 660 & 30 & 38 & 2.8 \\
B & 4.50 & 3000 & 38 & 30 & 10 \\
C & 8 & 1500 & 35 & 30 & 5 \\
D & 7 & 716 & 35 & 40 & 2.64 \\
E & 3 & 500 & 20 & 30 & No indica \\
F & 2 & 40 & 20 & 22 & 1.5 \\
G & 3 & $320^{*}$ & 27 & 50 & 2.8 \\
H & No indica & 400 & 32 & 30 & 2.5 \\
I & 8 & 1100 & 20 & \\
\hline
\end{tabular}

${ }^{\star}$ Dato extrapolado entre 74 abonados multiplicados por 3.5 personas por vivienda individual ocupada en promedio para Guanacaste (INEC, 2015).

El proceso de selección de las ASADAS participantes incluyó una consulta a la base de datos de la Oficina Regional de Acueductos Comunales (ORAC) del AyA, en los cantones con mayor afectación por la situación de sequía, durante los años 2014-2016. La estrategia de muestreo utilizada es no probabilística por autoselección. Se elaboró una lista y se consultó a todas las ASADAS de áreas afectadas por la sequía en el cantón de Nicoya sobre su disponibilidad de trabajar en el proceso. Posteriormente, se firmaron los consentimientos informados con todas las ASADAS participantes.

El número de casos elegidos no responde a ningún criterio probabilístico; sin embargo, proporciona información para evaluar las 9 ASADAS, con respecto al funcionamiento de estos acueductos. Los resultados, entonces, no pueden extrapolarse o considerarse representativos de toda la población de ASADAS del cantón nicoyano. Según el AyA, todas las ASADAS escogidas se encuentran en estado de vulnerabilidad a la sequía y se ubican en comunidades relativamente cercanas entre sí.

\subsection{Procedimiento de la evaluación}

La recolección de información clave sobre la gestión de las ASADAS se realizó aplicando un instrumento tipo encuesta denominado "Formulario unificado del Instituto Costarricense de Acueductos y Alcantarillados Subgerencia Gestión de Sistemas Comunales" (AyA, 2015). Este evalúa 5 componentes que las ASADAS deben cumplir en su gestión: (1) gestión administrativa y financiera, (2) gestión comercial, (3) gestión comunal, (4) gestión del recurso hídrico y (5) gestión de los sistemas de agua.

\begin{tabular}{|c|c|c|c|c|c|}
\hline 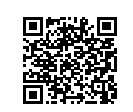 & (c) (i) (5) & $\underset{\text { AMBIENTALES }}{\Leftrightarrow}$ & $\frac{9 \%}{\text { euna }}$ & 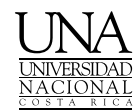 & 30 \\
\hline
\end{tabular}




\section{Revista de CIENCIAS AMBIENTALES Tropical Journal of Environmental Sciences}

Revista de Ciencias Ambientales (Trop J Environ Sci)

e-ISSN: 2215-3896

(Julio-Diciembre, 2019) . Vol 53(2): 25-46 DOI: https://doi.org/10.15359/rca.53-2.2

Open Access: www.revistas.una.ac.cr/ambientales e-mail: revista.ambientales@una.ac.cr Suárez A., Baldioceda A., Durán G., Rojas J., Rojas D., Guillén, A.

En la construcción del instrumento participaron diversas instituciones, entre las cuales están Universidad Nacional, Universidad de Costa Rica, Universidad Técnica Nacional, Ministerio de Salud, Servicio Nacional de Riego y Avenamiento (SENARA), Autoridad Reguladora de los Servicios Públicos (ARESEP) y Ministerio de Ambiente y Energía (MINAE).

El formulario unificado lo utiliza la Subgerencia de Gestión de Sistemas Comunales del AyA para evaluar las ASADAS del país en los componentes antes mencionados. Según la puntuación que la ASADA obtenga en cada uno de los componentes, se realiza una ponderación y se clasifican en las siguientes categorías: a) entre 80-100 puntos: consolidada; b) entre 60-79 puntos: desarrollo alto; c) entre 40-59 puntos: desarrollo bajo; d) menos de 40 puntos: desarrollo débil.

Al analizar las 101 preguntas del formulario, se identificó que es precisa una encuesta complementaria, a fin de obtener información sobre eventuales acciones implementadas por las ASADAS durante el periodo de sequía y para conocer la percepción sobre las principales necesidades de cada ASADA. Esta encuesta corta complementaria aporta datos prácticos de la gestión técnica de los sistemas de agua potable. Por ejemplo, se consultó si se aplicaron medidas de racionamiento de agua durante el periodo de sequía del 2014-2016 y cuáles son, según percepción personal, sus requerimientos primordiales.

Las encuestas fueron aplicadas a un integrante de la junta directiva, administrador o fontanero de la ASADA, con una duración promedio de una hora, durante el segundo semestre del 2017. Cuando una pregunta no pudo ser contestada, se obtuvo la información por medio de una segunda visita o llamada telefónica. No obstante, no todas las interrogantes pudieron ser siempre respondidas por los representantes de las ASADAS encuestadas. Por lo tanto, se toma en cuenta la variabilidad en cuanto a la disponibilidad de respuestas.

\subsection{Análisis fisicoquímicos y microbiológicos}

Los parámetros evaluados de calidad del agua potable incluyeron el nivel 1 ( $\mathrm{pH}$, conductividad eléctrica, turbiedad, color, sabor, olor y coliformes), según el Reglamento para la Calidad del Agua Potable (38924-S). Los muestreos se realizaron, principalmente, en la estación lluviosa (septiembre-octubre-noviembre), durante 3 años continuos (2016, 2017 y 2018). Resultó importante efectuarlos en dicha época, dado que las lluvias aumentan el riesgo de contaminación en los acueductos y pozos, debido a las posibles filtraciones de agua de lluvia con contaminantes orgánicos e inorgánicos del medio. Estos pueden llegar a los tanques y red de distribución, mediante fugas o roturas de los sistemas.

La toma de muestras se realizó en los pozos, los tanques y en 3 puntos de la red de distribución, siguiendo lo establecido en el Reglamento para la Calidad del Agua Potable (38924-S). Esas muestras se trasladaron a los laboratorios de calidad de agua del Centro de Recursos Hídricos para Centroamérica y el Caribe (HIDROCEC-UNA), Campus Liberia, Universidad Nacional.

Los análisis fisicoquímicos ( $\mathrm{pH}$, conductividad eléctrica, turbiedad, color, sabor, olor) fueron realizados basándose en el método 1060 (APHA, 2017). En el periodo 2016-2017, para los análisis microbiológicos se utilizó la prueba de tubos de fermentación múltiple para determinar

\begin{tabular}{|c|c|c|c|c|c|}
\hline 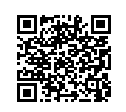 & (c) & $\underset{\text { AMBIENANALES }}{\infty}$ & $\frac{O \%}{20}$ & 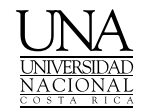 & 31 \\
\hline
\end{tabular}




\section{Revista de CIENCIAS AMBIENTALES Tropical Journal of Environmental Sciences}

Revista de Ciencias Ambientales (Trop J Environ Sci)

e-ISSN: 2215-3896

(Julio-Diciembre, 2019) . Vol 53(2): 25-46 DOI: https://doi.org/10.15359/rca.53-2.2

Open Access: www.revistas.una.ac.cr/ambientales e-mail: revista.ambientales@una.ac.cr Suárez A., Baldioceda A., Durán G., Rojas J., Rojas D., Guillén, A.

Escherichia coli, a partir del método $9221 \mathrm{~F}$, y coliformes fecales, siguiendo el Método $9221 \mathrm{E}$ (APHA, 2017). En el 2017, se empleó la prueba de Colilert®-18/Quanti-Tray® de IDEXX, que sustituye al método de los tubos múltiples, como nueva norma internacional para la detección de coliformes totales y E. coli en el agua. El cambio obedece a que la Organización Mundial de Normalización ha retirado la norma ISO 9308-2:1990, que recomendaba el método de los tubos múltiples (número más probable), y la ha sustituido por la norma ISO 9308-2:2012, que sugiere el método Colilert-18/Quanti-Tray, dado que se considera una detección fácil, rápida y mucho más precisa de coliformes y $E$. coli que el método de tubos de fermentación múltiple.

\section{Resultados}

Los resultados obtenidos de la aplicación del formulario unificado permitieron determinar el grado de fragilidad en el que se encuentran las ASADAS estudiadas. Únicamente la ASADA B clasificó en la categoría de desarrollo alto, según la clasificación de la organización comunal prestadora de servicios de agua potable y saneamiento (Cuadro 2), el resto se catalogó como ASADAS con desarrollo bajo y débil.

Según los 5 componentes evaluados de la gestión del agua (gestión administrativa y financiera, gestión comercial, gestión comunal, gestión del recurso hídrico y gestión de los sistemas de agua), se obtuvo un débil manejo en 5 de las 9 ASADAS evaluadas (A, E, F, G y H), más 3 con desarrollo bajo (C, D e I).

Cuadro 2. Resultados de la evaluación de los acueductos rurales, de acuerdo con el formulario unificado de información sobre organizaciones comunales prestadoras de servicios de agua potable y saneamiento

\begin{tabular}{ccc}
\hline ASADA & Puntaje total & Descripción de la categoría ${ }^{\star}$ \\
\hline A & 36.50 & Débil \\
B & 61.00 & Desarrollo alto \\
C & 51.50 & Desarrollo bajo \\
D & 46.33 & Desarrollo bajo \\
E & 25.50 & Débil \\
F & 26.00 & Débil \\
G & 20.00 & Débil \\
H & 34.50 & Débil \\
I & 44.50 & Desarrollo bajo \\
\hline
\end{tabular}

* Categorías: a) 80-100 puntos: consolidada; b) 60-79 puntos: desarrollo alto; c) 40-59 puntos: desarrollo bajo; d) menos de 40 puntos: débil.

La Figura 2 muestra en una matriz las respuestas obtenidas de la sección de información básica del formulario unificado, datos relacionados, principalmente, con la operación legal de las ASADAS. Cada columna representa una ASADA; las filas, las distintas preguntas; las tonalidades de grises indican la respuesta de la ASADA a cada pregunta (no se respondió a las preguntas de

\begin{tabular}{|c|c|c|c|c|c|}
\hline 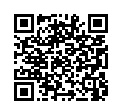 & (c) & $\overbrace{\text { AMBIENTIIIS }}$ & $\frac{O O}{2 \%}$ & $\frac{\text { UNA }}{\frac{\text { UNIVERSIDAD }}{\text { NACIONAL }}}$ & 32 \\
\hline
\end{tabular}




\section{Revista de CIENCIAS AMBIENTALES Tropical Journal of Environmental Sciences}

Revista de Ciencias Ambientales (Trop J Environ Sci)

e-ISSN: $2215-3896$

(Julio-Diciembre, 2019) . Vol 53(2): 25-46

DOI: https://doi.org/10.15359/rca.53-2.2

Open Access: www.revistas.una.ac.cr/ambientales e-mail: revista.ambientales@una.ac.cr

Suárez A., Baldioceda A., Durán G., Rojas J., Rojas D., Guillén, A.

la ASADA H para esta sección). Únicamente 5 participantes respondieron que cuentan con un convenio de delegación, necesario para operar formalmente. De las ASADAS valoradas, solo 2 colocaron hidrantes en su comunidad; este aspecto es de gran importancia para la prevención de incendios, sobre todo en una región donde durante época seca aumentan los eventos naturales y provocados que ocasionan incendios forestales. Se pudo identificar un bajo nivel de conocimiento sobre los costos reales y de mantenimiento de los acueductos, por parte de los integrantes de las ASADAS. Otro aspecto identificado como importante fue que solamente 2 ASADAS tienen macromedidores para cuantificar el volumen de agua extraído del pozo. El contar con macromedición es muy importante para llevar un registro del agua no contabilizada, esa que podría estarse perdiendo por fugas o conexiones ilegales. El problema con la existencia de fugas es que son puntos de riesgo para la entrada de patógenos al sistema de distribución de agua potable. No obstante lo anterior, la micromedición sí se aplica en todas las ASADAS.

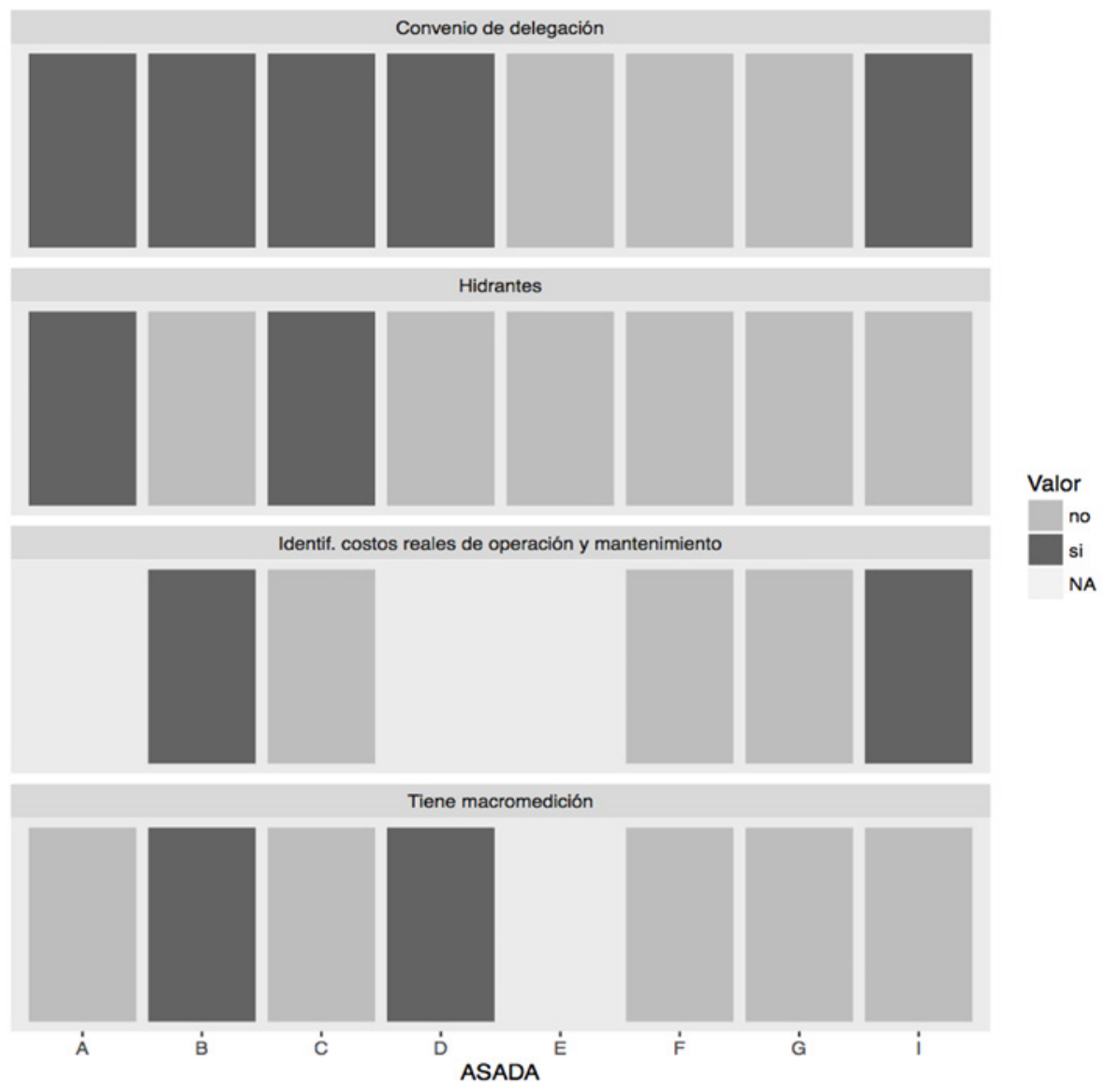

Figura 2. Información general básica para el funcionamiento legal de una ASADA. Se muestran las respuestas dicotómicas obtenidas de la consulta sobre presencia de hidrantes, convenio de delegación, conocimiento de costos de operación y mantenimiento, al igual que macromedición en las ASADAS evaluadas.

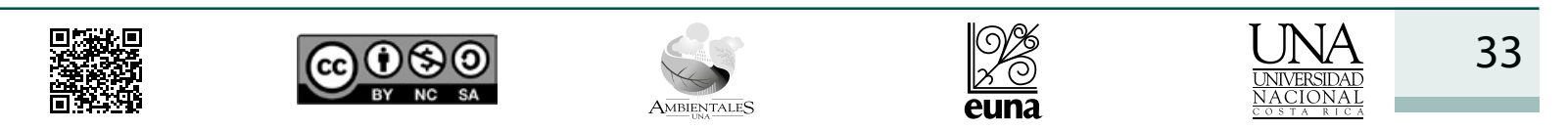




\section{Revista de CIENCIAS AMBIENTALES Tropical Journal of Environmental Sciences}

Revista de Ciencias Ambientales (Trop J Environ Sci) e-ISSN: 2215-3896

(Julio-Diciembre, 2019) . Vol 53(2): 25-46 DOI: https://doi.org/10.15359/rca.53-2.2

Open Access: www.revistas.una.ac.cr/ambientales e-mail: revista.ambientales@una.ac.cr Suárez A., Baldioceda A., Durán G., Rojas J., Rojas D., Guillén, A.

La información que se muestra en la Figura 3 también se obtuvo del análisis de las respuestas surgidas del formulario unificado aplicado. Igual que la Figura 2, muestra en cada columna una ASADA y, en las filas, los valores consultados (en este caso tampoco se obtuvo respuesta de la ASADA H). La tasa anual de expansión más alta es en las ASADAS C y G, a pesar de que la $\mathrm{G}$ no aportó un dato exacto de población abastecida (Figura 3). La mayoría de las ASADAS posee solo 1 pozo y 1 tanque de almacenamiento para abastecer a la población; únicamente la ASADA I tiene 4 pozos, de los cuales 2 se secaron durante el periodo de sequía, por lo que fueron retirados de funcionamiento. La capacidad de los tanques de almacenamiento tuvo un rango desde $9000 \mathrm{~m}^{3}$ hasta $206000 \mathrm{~m}^{3}$, siendo la ASADA B la que cuenta con una mayor capacidad de almacenamiento de agua potable.

Como resultado de la encuesta complementaria, con respecto a las acciones de adaptación durante el periodo de sequía, se obtuvo que únicamente la ASADA I realizó racionamientos de 5 horas o más. Cuando se consultó sobre las prioridades dentro del acueducto, estas siempre estuvieron relacionadas con la disponibilidad de agua (perforación de un nuevo pozo, otro tanque o uno de mayor capacidad). En segundo lugar, los participantes describieron una mayor capacidad de almacenamiento (o cambio de tuberías) y en tercer plano las respuestas fueron variadas, incluyendo: equipo de cloración, hidrantes, recursos económicos, nuevamente tanques de almacenamiento.

La Figura 4 muestra las brechas en el funcionamiento de las ASADAS evaluadas, con respecto a su gestión administrativa-financiera, la de sistemas de agua potable, la del recurso hídrico, la comunal y la comercial, según el formulario unificado aplicado. La Figura 4 denota en detalle cuáles de los componentes de las ASADAS consiguieron mayor o menor porcentaje. El rango del puntaje total obtenido por ASADA estuvo entre 20 y $61 \%$ del total obtenido.

En la Figura 5, se observan los resultados de la evaluación de la calidad del agua, por medio de los análisis fisicoquímicos, y, en la Figura 6, los resultados de los análisis microbiológicos realizados. En las columnas, se muestran las distintas ASADAS y, en las filas, los parámetros analizados. El color representa el sitio donde se tomó la muestra y la forma, el año muestreado. En el caso de los análisis fisicoquímicos, también se agregaron gráficos de caja para recalcar la distribución de las mediciones. Los resultados permitieron identificar debilidades en la parte operativa de las ASADAS de la gestión del agua.

En la Figura 5, se observa los valores de cloro libre ( $\mathrm{mg} / \mathrm{l})$ obtenidos, que, de acuerdo con el Reglamento para agua potable 38924-S, deben tener un nivel aceptable entre 0.2-0.6 \pm 0.01 $\mathrm{mg} / \mathrm{l}$. No obstante, se apreció que, en la mayoría de las ASADAS, los datos de cloro libre fueron cercanos a cero, en pozo, tanque o red de distribución, lo cual representa un riesgo, si existe entrada de patógenos a la red de distribución. Por otra parte, la ASADA I presentó un valor muy alto con respecto al permisible $(2.5 \mathrm{mg} / \mathrm{l})$, en uno de sus pozos; sin embargo, las demás mediciones en el tanque y red de distribución sí se encontraron en los rangos permitidos; este mismo comportamiento lo tuvo ASADA B.

\begin{tabular}{|c|c|c|c|c|}
\hline 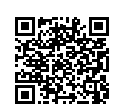 & (c) (i) (-) & $\underset{\text { AMBIENALIES }}{\longrightarrow}$ & $\frac{\sigma \%}{\text { euna }}$ & 34 \\
\hline
\end{tabular}


Revista de

CIENCIAS AMBIENTALES

Tropical Journal of Environmental Sciences
Revista de Ciencias Ambientales (Trop J Environ Sci)

e-ISSN: 2215-3896

(Julio-Diciembre, 2019) . Vol 53(2): 25-46

DOI: https://doi.org/10.15359/rca.53-2.2

Open Access: www.revistas.una.ac.cr/ambientales

e-mail: revista.ambientales@una.ac.cr Suárez A., Baldioceda A., Durán G., Rojas J., Rojas D., Guillén, A.

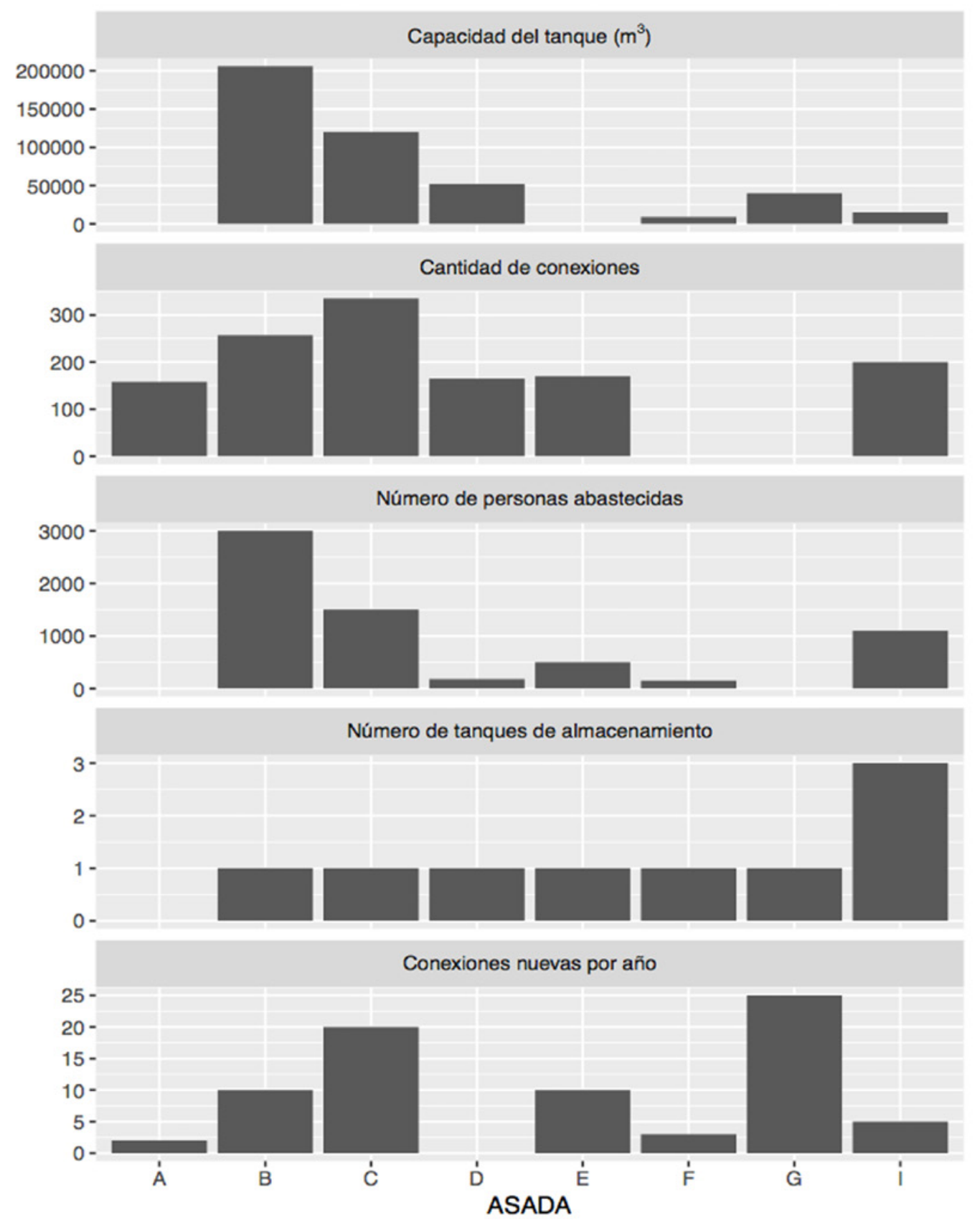

Figura 3. Información obtenida de las ASADAS evaluadas sobre tasa anual de expansión, cantidad de tanques de almacenamiento, población abastecida, conexiones y capacidad de almacenamiento de los tanques de agua.

\begin{tabular}{|c|c|}
\hline 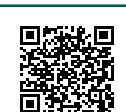 & (c) (i) (으 () \\
\hline
\end{tabular}




\section{Revista de CIENCIAS AMBIENTALES Tropical Journal of Environmental Sciences}

Revista de Ciencias Ambientales (Trop J Environ Sci)

e-ISSN: 2215-3896

(Julio-Diciembre, 2019) . Vol 53(2): 25-46

DOI: https://doi.org/10.15359/rca.53-2.2

Open Access: www.revistas.una.ac.cr/ambientales

e-mail: revista.ambientales@una.ac.cr Suárez A., Baldioceda A., Durán G., Rojas J., Rojas D., Guillén, A.
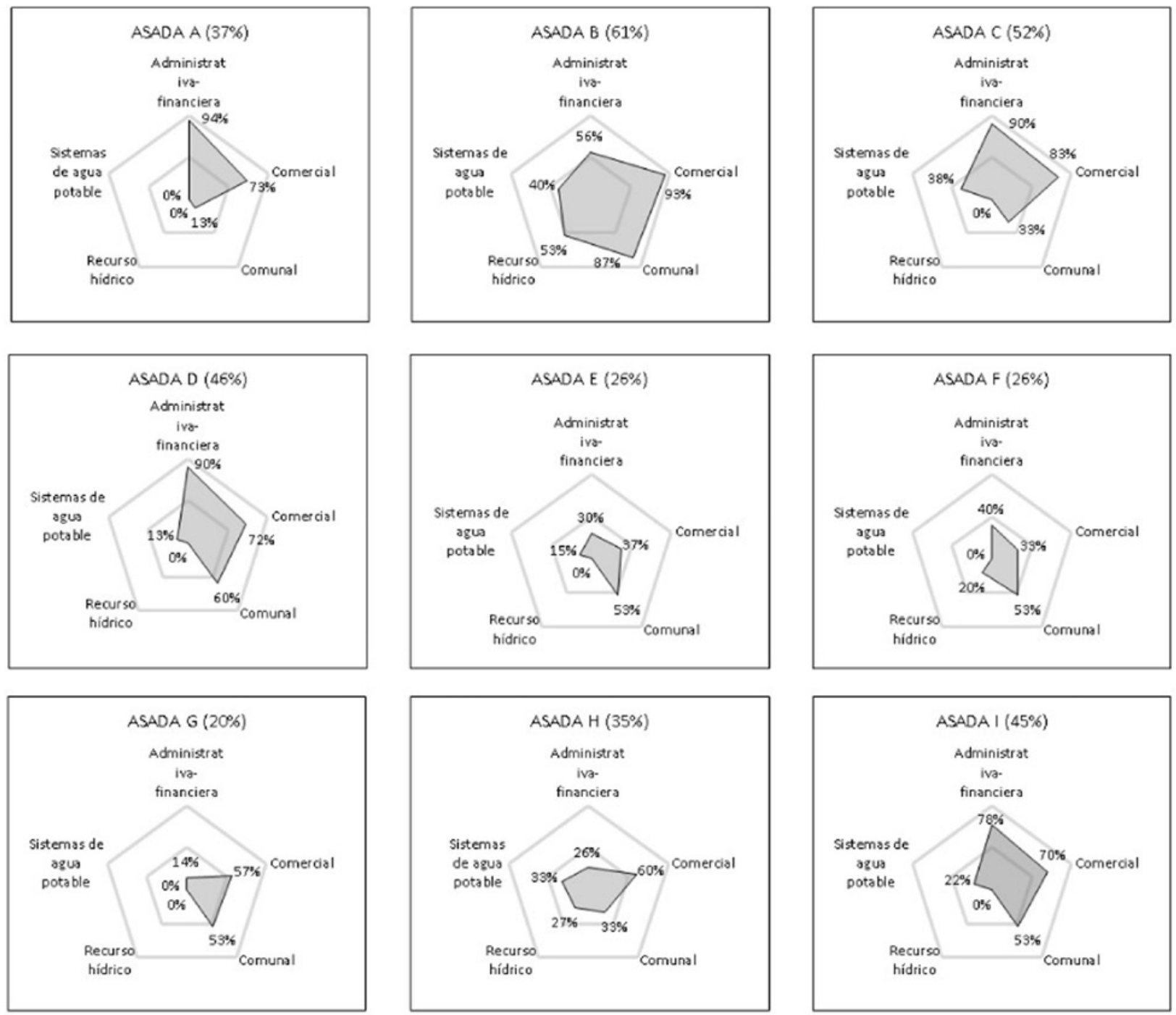

Figura 4. Brechas en el funcionamiento de las ASADAS con respecto a los componentes de gestión evaluados en el formulario unificado. Entre paréntesis, se muestra, en cada telaraña, el porcentaje obtenido del puntaje total y los pentágonos indican los porcentajes obtenidos para cada componente.

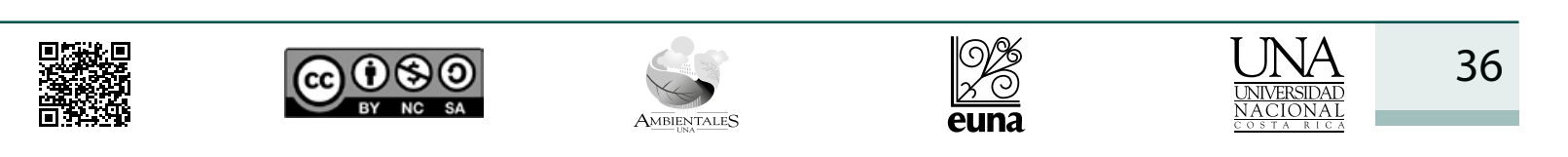




\section{Revista de CIENCIAS AMBIENTALES Tropical Journal of Environmental Sciences}

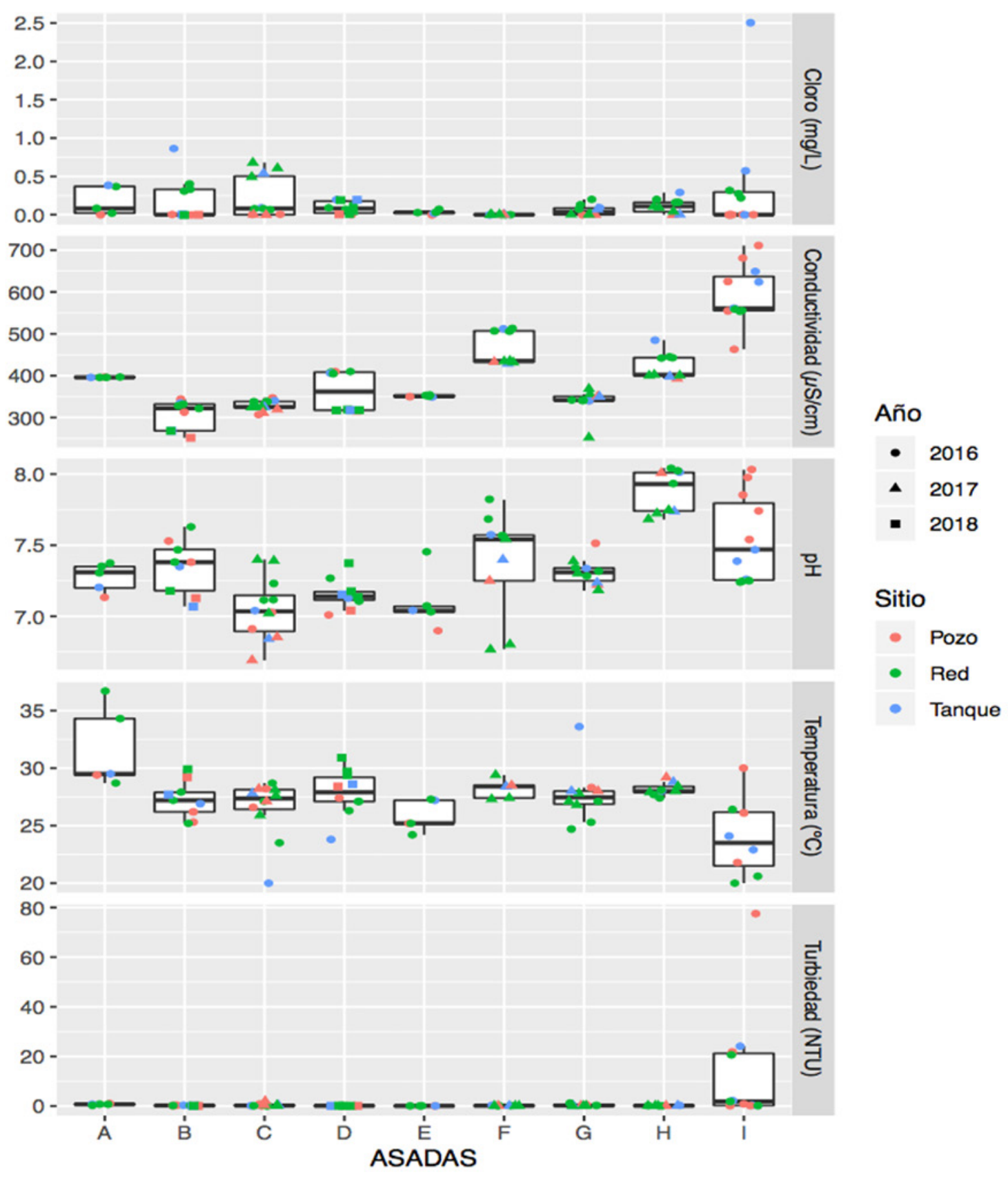

Figura 5. Parámetros fisicoquímicos analizados en muestras de agua para consumo humano tomadas en muestras del pozo, de la red de distribución y del tanque de almacenamiento de 9 ASADAS del cantón de Nicoya, Guanacaste, Costa Rica.

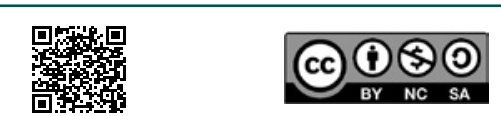




\section{Revista de CIENCIAS AMBIENTALES Tropical Journal of Environmental Sciences}

Revista de Ciencias Ambientales (Trop J Environ Sci)

e-ISSN: 2215-3896

(Julio-Diciembre, 2019) . Vol 53(2): 25-46 DOI: https://doi.org/10.15359/rca.53-2.2

Open Access: www.revistas.una.ac.cr/ambientales e-mail: revista.ambientales@una.ac.cr Suárez A., Baldioceda A., Durán G., Rojas J., Rojas D., Guillén, A.

Las ASADAS I, H y F mostraron valores por arriba del máximo permitido de conductividad y $\mathrm{pH}$, asociados, probablemente, al tipo de sales disueltas en el agua, relacionadas con la geología de la zona. Las ASADAS I y F presentaron valores por encima del permitido, en cuanto a conductividad ( $>400 \pm 1 \mu \mathrm{S} / \mathrm{cm}$ ); esta es producida por los electrolitos disueltos en agua, lo cual concuerda con que en I se obtuviera los valores más altos de turbidez. La Organización Mundial de la Salud y la legislación nacional indican un valor de referencia de 5 NTU para agua potable (Reglamento para la Calidad del Agua Potable, 38924-S). Se encontraron datos de turbidez en el rango aceptable; solamente en la ASADA I se obtuvieron valores fuera de ese rango, causados por partículas en suspensión de sedimento, materia orgánica y suelo, posiblemente arrastrados de material erosionado por las lluvias de la época, cuando se realizó el muestreo.

La Figura 6 señala los resultados de los análisis microbiológicos de coliformes totales, fecales y Escherichia coli; el reglamento indica que no debe detectarse presencia de estos microorganismos (No detectable NMP/100 ml). No obstante, se detectaron microorganismos tanto en el pozo como en la red de distribución, en las ASADAS C, F e I.

\section{Discusión}

Las ASADAS evaluadas poseen un grado de fragilidad alto. Solamente 1 de ellas presentó desarrollo alto en su nivel de gestión; 3 en bajo, y 5, una gestión débil. El bajo rendimiento en el funcionamiento, en el nivel global, se pudo determinar al ponderar los resultados obtenidos del formulario unificado, en el cual se obtuvo que las ASADAS tienen un bajo desempeño en los 5 componentes evaluados, en lo organizativo-administrativo, la gestión de sus sistemas y sus fuentes. Por ejemplo, el hecho de que no cuentan con macromedición en sus pozos es algo fundamental para saber cuánta agua extraen de ellos y, por lo tanto, para conocer el agua no contabilizada. El uso del agua en una ASADA puede ser más eficiente, si se reduce el porcentaje de la no contabilizada, porque esto quiere decir que el sistema se encuentra en buen estado, y se podría identificar también si existen conexiones ilegales. Otro de los factores importantes que condicionaron el grado de desarrollo de las ASADAS fue el hecho de que no cuentan con saneamiento ni alcantarillado adecuado.

Durante las entrevistas, los integrantes de las ASADAS mencionan, en reiteradas ocasiones, una falta de participación comunal en temas referentes a la protección y al uso racional del agua en las distintas zonas. Lo anterior concuerda con lo estudiado por Madrigal-Ballestero y Naranjo (2015), quienes observaron que factores como la estructura de gobernanza y sus interacciones determinan marcadas diferencias en el rendimiento. Ellos encontraron que los factores más relevantes incluyen la infraestructura para el suministro de agua, el capital humano del comité de agua, las reglas financieras y operativas de la organización, así como los mecanismos de rendición de cuentas; tales aspectos fueron débiles en la mayoría de las ASADAS evaluadas.

La vulnerabilidad de las ASADAS en el cantón de Nicoya, ante eventos hidrometereológicos extremos (secos y húmedos), es alta, principalmente, por la antigüedad de los sistemas, la falta de mantenimiento y la poca capacidad de sus tanques de almacenamiento. Por lo tanto,

\begin{tabular}{|c|c|c|c|c|c|}
\hline 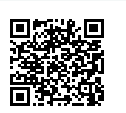 & (c) (1) $(9)$ & $\underset{\text { AMBIENTAIES }}{\longrightarrow}$ & $\frac{O O \%}{2 \frac{O}{2}}$ & $\frac{\text { UNA }}{\frac{\text { UNIVERSIDAD }}{\text { NACIONAL }}}$ & 38 \\
\hline
\end{tabular}




\section{Revista de CIENCIAS AMBIENTALES Tropical Journal of Environmental Sciences}

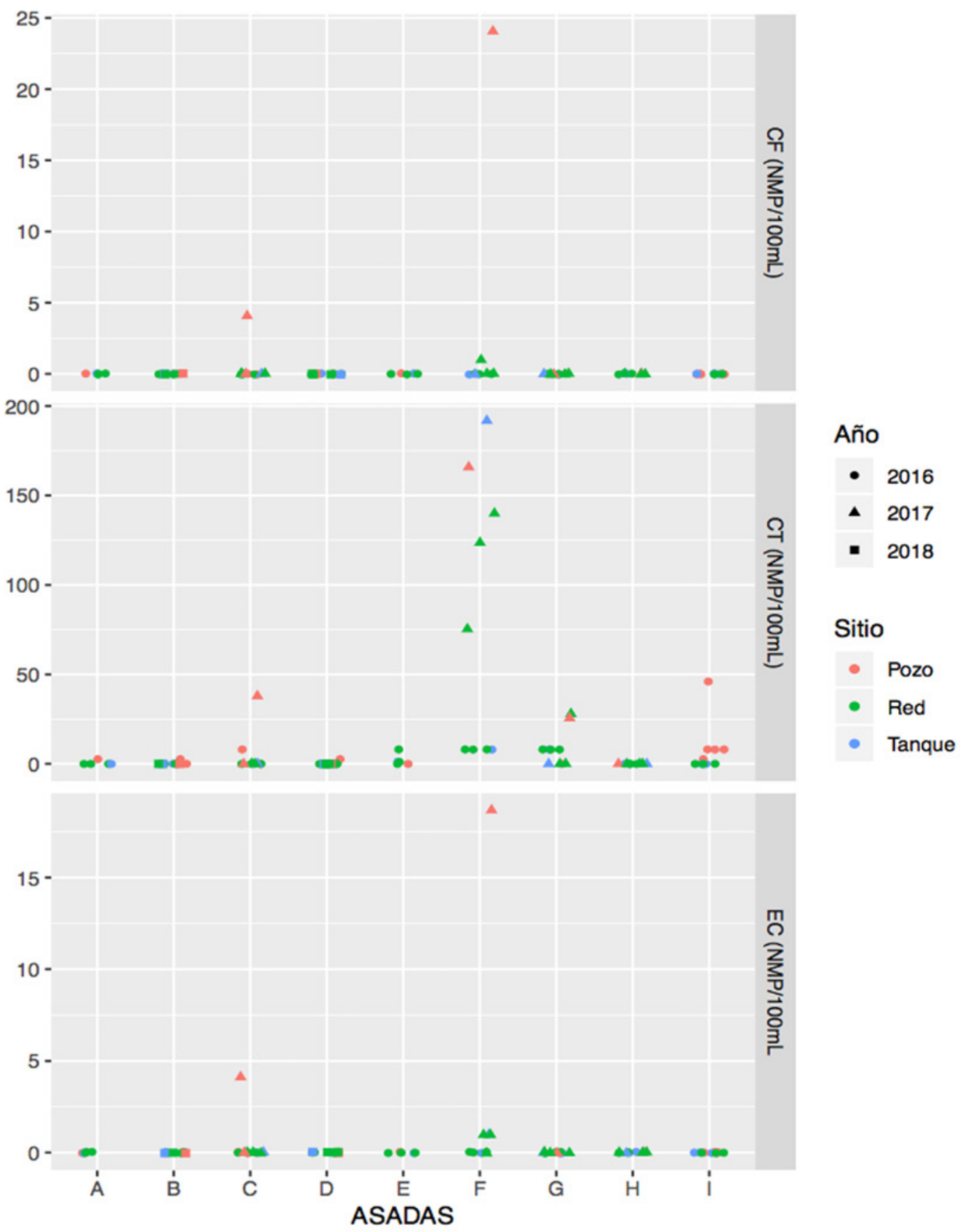

Figura 6. Parámetros microbiológicos (coliformes totales, coliformes fecales y E.coli) en muestras de agua para consumo humano, tomadas en muestras del pozo, de la red de distribución y del tanque de almacenamiento de 9 ASADAS del cantón de Nicoya, Guanacaste, Costa Rica (2016-2018).

(cc)

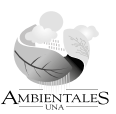




\section{Revista de CIENCIAS AMBIENTALES Tropical Journal of Environmental Sciences}

Revista de Ciencias Ambientales (Trop J Environ Sci)

e-ISSN: 2215-3896

(Julio-Diciembre, 2019) . Vol 53(2): 25-46 DOI: https://doi.org/10.15359/rca.53-2.2

Open Access: www.revistas.una.ac.cr/ambientales e-mail: revista.ambientales@una.ac.cr Suárez A., Baldioceda A., Durán G., Rojas J., Rojas D., Guillén, A.

podrían representar un riesgo creciente, ante escenarios de cambio y variabilidad climática previstos para la provincia de Guanacaste. Los factores de riesgo de escasez, calidad inadecuada para el consumo humano y daños por inundaciones observados atentan contra los niveles aceptables de seguridad hídrica para las comunidades rurales (OECD, 2013).

El modelo de delegación de suministro de agua potable y saneamiento implementado en Costa Rica tiene un enfoque de demanda. De acuerdo con Moriarty, Smits, Butterworth y Franceys (2013), este es el enfoque dominante del suministro de agua rural en los países en desarrollo, lo cual ha dado como resultado un bajo rendimiento de los proveedores de servicios, las altas tasas de fallas del sistema y niveles muy bajos de servicio. Sin embargo, es importante resaltar que las organizaciones comunitarias de agua potable como las ASADAS, en los niveles nacional y latinoamericano, son los proveedores más importantes de agua en áreas rurales y juegan un papel clave tanto en la descentralización como en los procesos de democratización del acceso al agua para consumo humano (Astorga, 2016).

De acuerdo con Montgomery, Bartram y Elimelech (2009), existen 3 componentes de sostenibilidad universales, que conducen, a largo plazo, al buen funcionamiento de suministros de agua y saneamiento, los cuales son: la demanda efectiva de la comunidad, el financiamiento local y la recuperación del costo, lo que permite una operación dinámica y el mantenimiento adecuado. Estos autores también han reconocido algunos factores importantes para hacer efectivos cada uno de dichos componentes de sostenibilidad, por ejemplo: la planificación participativa, la elección de tecnología apropiada y la comercialización, bajo esquemas de préstamo y ahorro, planificación financiera, así como bajo financiamiento proveniente de diferentes fuentes. En este estudio, se identificó la necesidad que tienen los integrantes de las ASADAS de mejorar en esos 3 componentes, para garantizar la sostenibilidad de los sistemas de abastecimiento de agua potable.

Las encuestas y visitas a las ASADAS fueron actividades con las cuales se logró evidenciar que estos grupos de personas que las conforman requieren mucho acompañamiento y capacitación por parte de las instituciones públicas, para que puedan tener acceso a fuentes de financiamiento y fortalecer su gestión. También, se pudo apreciar que se requiere colaboración de los pobladores beneficiados, con el propósito de proteger el recurso de la contaminación y el desperdicio. El suministro de agua rural descentralizado requiere roles, habilidades, estrategias y disposiciones estructurales bien definidas tanto en el nivel nacional como donde se administra y utiliza el servicio (Alfaro, 2018).

En las ASADAS, existe la estructura organizativa que se rige por la Ley de Asociaciones 218. La función de la Junta Directiva es un trabajo ad honorem; si la ASADA cuenta con un poco de liquidez, contrata a un administrador y servicios de fontanería. Sin embargo, muchas veces esos roles no están muy bien definidos y un presidente o algún otro integrante de la ASADA realiza muchas funciones sin reconocimiento. Se ha visto en otras latitudes que la gestión comunitaria como modelo no ha fallado, sino que está llegando a los límites de lo que se puede lograr de manera realista, bajo un enfoque basado en la informalidad y el voluntarismo (Sandoval-Moreno y Günther, 2013).

\begin{tabular}{|c|c|c|}
\hline 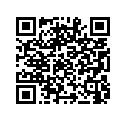 & (c) & 40 \\
\hline
\end{tabular}




\section{Revista de CIENCIAS AMBIENTALES Tropical Journal of Environmental Sciences}

Revista de Ciencias Ambientales (Trop J Environ Sci)

e-ISSN: 2215-3896

(Julio-Diciembre, 2019) . Vol 53(2): 25-46 DOI: https://doi.org/10.15359/rca.53-2.2

Open Access: www.revistas.una.ac.cr/ambientales e-mail: revista.ambientales@una.ac.cr Suárez A., Baldioceda A., Durán G., Rojas J., Rojas D., Guillén, A.

Con respecto a los análisis físicos-químicos medidos en las ASADAS del estudio, se pudo observar que, en la mayoría, las conductividades se mantienen en el rango aceptable exigido por el Reglamento para la Calidad del Agua Potable $(400 \mu / \mathrm{cm})$, a excepción de las ASADAS I, $\mathrm{H}$ y F, las cuales muestran valores de $\mathrm{pH}$ más elevados. Una mayor profundidad del nivel freático podría implicar más conductividad del agua, debido a los tiempos de contacto que el agua profunda mantiene con las rocas del acuífero. Así, la obtención de valores de conductividad por encima de lo establecido en el agua de los pozos podría estar relacionada con la composición geológica del acuífero en época seca. No obstante, esos valores pueden ser modificados, dados los procesos de infiltración de aguas superficiales durante la época lluviosa, lo que conduce a una posible dilución de las sales y un mayor aporte de ácido carbónico $\left(\mathrm{H}_{2} \mathrm{CO}_{3}\right)$, proveniente de la reacción del $\mathrm{CO}_{2}$ con el agua de lluvia (Custodio y Llamas, 1996).

Durante la época de lluvias, la calidad del agua de los acuíferos libres puede ser afectada por los contaminantes disueltos en las aguas superficiales de infiltración reciente. Por ello, a menor profundidad del nivel freático, más vulnerable es el recurso a la contaminación (Custodio y Llamas, 1996).

Las ASADAS estudiadas no realizan cloración en el pozo, por lo que sus niveles de cloro libre no son detectables. La cloración se lleva a cabo en el tanque, en su mayoría, a través de un sistema de pastillas. Sin embargo, del total de las ASADAS estudiadas, únicamente la A, C e I cumplieron con el parámetro durante los muestreos del periodo de análisis 2016-2017 (0.2-0.6 $\mathrm{mg} / \mathrm{l}$ cloro residual libre). Esta situación tiene una importante relación con los datos obtenidos de los exámenes microbiológicos, en los cuales se observa que, durante los muestreos, las ASADAS E, G y, principalmente, F, muestran contenidos importantes de coliformes totales durante la época lluviosa.

La identificación de microorganismos en las muestras de agua evaluadas en el pozo, en el tanque y en la red de distribución implica un riesgo para la seguridad hídrica, pues la ASADA que presentó mayor número de coliformes fecales y E. coli no utilizaba clorador para el tratamiento del agua de consumo. La contaminación de aguas de consumo con materia fecal de ganado vacuno, así como con aguas negras o materia fecal humana se ha asociado con brotes de diarrea. Sin embargo, es importante resaltar que no solamente bacterias pueden ingresar al sistema. Luna, Reyes, Chinchilla, y Catarinella (2002) investigaron la presencia del protozoario Cryptosporidium en aguas superficiales de agua de consumo, tratadas por los métodos usuales, como la desinfección química o los procedimientos de filtración, y encontraron que no son eficientes en la remoción de los ooquistes de este protozoario, el cual es una causa de diarrea infantil en Costa Rica.

Es importante tomar en cuenta que, globalmente, cerca del $10 \%$ del total de la carga de enfermedades está relacionado con el agua no potable, el saneamiento y la higiene, los cuales cobran 3.6 millones de vidas anualmente (Prüss-Üstün, Gore, Bartram y Bos, 2008). Costa Rica tiene una buena cobertura de los sistemas de salud, lo que posiblemente colabore en la atención de enfermos por padecimientos vinculados con el agua, pero no existen estudios

\begin{tabular}{|c|c|c|c|c|c|}
\hline 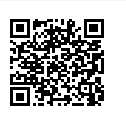 & (c) (1) $(9)$ & $\underset{\text { AMBIENTAIES }}{\longrightarrow}$ & $\frac{O O \%}{2 \frac{O}{2}}$ & $\frac{\text { UNA }}{\frac{\text { UNIVERSIDAD }}{\text { NACIONAL }}}$ & 41 \\
\hline
\end{tabular}




\section{Revista de CIENCIAS AMBIENTALES Tropical Journal of Environmental Sciences}

Revista de Ciencias Ambientales (Trop J Environ Sci)

e-ISSN: 2215-3896

(Julio-Diciembre, 2019) . Vol 53(2): 25-46 DOI: https://doi.org/10.15359/rca.53-2.2

Open Access: www.revistas.una.ac.cr/ambientales e-mail: revista.ambientales@una.ac.cr

Suárez A., Baldioceda A., Durán G., Rojas J., Rojas D., Guillén, A.

epidemiológicos recientes que evalúen la incidencia en enfermedades transmitidas por el agua y la gestión de los sistemas de distribución de agua potable.

Chamizo y Mora (2006) realizaron un estudio sobre los procesos de contaminación del agua y el suelo, como resultado de deficiencias en la gestión ambiental, las cuales tienen consecuencias graves en la salud de las personas. Ellos diseñaron un estudio epidemiológico tipo ecológico utilizando información sanitaria y ambiental secundaria. Sin embargo, no se han llevado a cabo estudios más detallados en la zona de estudio ni en el nivel nacional. Contar con información de este tipo es fundamental, pues el acceso a agua y saneamiento mejorados es la piedra angular de comunidades saludables y juega un papel importante para mantener las ganancias de salud, económicas y sociales (Montgomery et al., 2009).

Una forma efectiva para garantizar sistemáticamente la seguridad de un mecanismo de abastecimiento de agua de consumo humano es aplicando un abordaje integral de evaluación y gestión de los riesgos, que abarque todas las etapas del proceso de abastecimiento de agua, desde la captación hasta que llega al consumidor. Esta metodología se denomina planes de seguridad del agua (PSA). Los principales objetivos de un PSA para garantizar la aplicación de buenas prácticas de abastecimiento de agua de consumo humano son la prevención o minimización de la contaminación en las fuentes; la reducción o eliminación de esa contaminación, a través de procesos de tratamiento; la prevención de tal contaminación durante el almacenamiento; la distribución y la gestión del agua de consumo humano (World Health Organization, 2012).

La problemática relacionada con el agua para el consumo humano en comunidades rurales de la provincia de Guanacaste es muy diversa. En los últimos años, se han presentado situaciones como la presencia de arsénico (Mora y Vargas, 2013), la sequía enlazada al fenómeno ENOS y actividades humanas que han contribuido tanto con el deterioro de la calidad como de la cantidad del recurso hídrico. Entre estas últimas, se halla la sobreexplotación de acuíferos costeros que provoca la intrusión salina; desarrollos inmobiliarios sin procedimientos adecuados; infraestructura hídrica obsoleta y sin mantenimiento adecuado, al igual que un poco o escaso acompañamiento estatal.

El AyA no posee los recursos para atender todas las necesidades de capacitación y monitoreo de la calidad del agua, por ello, delega el manejo del agua potable y el saneamiento a más de 1400 ASADAS en todo el país. Tomando en cuenta la problemática relacionada con aspectos legales, técnicos, económicos, ambientales y administrativos con los que lidian las ASADAS, la asociatividad es un proceso institucional que cada vez toma mayor importancia entre las ASADAS, porque, además, permite articulación, intercambio, comunicación y coordinación entre estas en los niveles de localidad, región o país. El fin de este proceso es aprender y fortalecer sus capacidades de gestión, incidencia en políticas públicas e innovación, de manera sostenible, orientadas al propósito común de proveer mayor y mejor acceso al agua a las comunidades (AyA, 2015).

Esta asociatividad puede darse a través de la constitución de federaciones, ligas o uniones y se les denomina (FLU). En Guanacaste, a la fecha hay conformadas las siguientes de ASADAS:

\begin{tabular}{|c|c|c|}
\hline 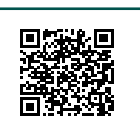 & (c) (i) @) (2) & 42 \\
\hline
\end{tabular}




\section{Revista de CIENCIAS AMBIENTALES Tropical Journal of Environmental Sciences}

Revista de Ciencias Ambientales (Trop J Environ Sci)

e-ISSN: 2215-3896

(Julio-Diciembre, 2019) . Vol 53(2): 25-46 DOI: https://doi.org/10.15359/rca.53-2.2

Open Access: www.revistas.una.ac.cr/ambientales e-mail: revista.ambientales@una.ac.cr Suárez A., Baldioceda A., Durán G., Rojas J., Rojas D., Guillén, A.

Liga Comunal del Agua Nicoya, Hojancha, Nandayure; Federación de ASADAS Liberia-La Cruz; Unión de ASADAS costeras de Santa Cruz; Federación de ASADAS Carrillo y Santa Cruz; Federación de ASADAS del territorio de Abangares, Cañas, Tilarán y Bagaces.

La organización de FLU en Guanacaste como agrupaciones de segundo nivel puede ser positiva para integrar el trabajo que realizan las ASADAS en su territorio, por ejemplo, en el nivel de zona geográfica. Al mismo tiempo, sirven como plataformas que implementan medidas de adaptación al cambio climático, con un enfoque territorial que incluya la gestión del riesgo, ante eventos hidrometereológicos extremos. Es importante reiterar que la gestión comunitaria requiere apoyo institucional continuo, porque las políticas y las tecnologías cambian, las personas se mueven dentro de las organizaciones comunales cada 2 años y el conocimiento técnico también puede volverse obsoleto.

\section{Conclusiones}

Las ASADAS evaluadas no están siendo gestionadas de manera adecuada. Por consiguiente, el agua que se distribuye a las comunidades compromete su seguridad hídrica, porque no siempre está disponible cuando se necesita y puede no cumplir con algunos de los parámetros básicos de potabilidad.

Los sistemas distribuidores de agua potable y saneamiento, por su desarrollo principalmente bajo y en condición frágil, son altamente vulnerables a los eventos hidrometereológicos que puedan ocurrir en el territorio. Esto atenta contra la disponibilidad acuífera tanto en época de sequía como durante inundaciones, considerando la poca capacidad de almacenamiento de agua potable. Además, la falta de mantenimiento de los sistemas y la antigüedad de los pozos podrían ser un riesgo para la posible entrada de agentes contaminantes a la red de distribución.

Es importante tomar en cuenta que las ASADAS estudiadas de la región Chorotega, por sus particularidades, necesitan acompañamiento y un abordaje integral, basado en las necesidades de cada una de ellas y sus componentes universales de sostenibilidad. Además, requieren capacitaciones participativas en temas primordiales como aspectos legales, administrativos, de calidad y cantidad de agua y sobre la vulnerabilidad en el manejo de las aguas. De aquí, lo fundamental de promover el uso de metodologías como los planes de seguridad del agua (PSA), al igual que de fortalecer el trabajo territorial y en organizaciones de segundo nivel, las cuales permitan fortalecer la gestión comunitaria hacia la seguridad hídrica.

La creación de federaciones, ligas o uniones (FLU) surge como un escenario interesante para trabajar articuladamente con grupos organizados de ASADAS que buscan un objetivo común de mejora de la gestión de los sistemas de abastecimiento de agua potable y saneamiento. Las FLU pueden ser, además, plataformas para abordar la adaptación al cambio climático, de manera que puedan participar en el monitoreo de sus fuentes de agua, con el fin de generar información y una mejor comprensión tanto de las amenazas como de los riesgos a los cuales están expuestas, esos que podrían incrementarse a futuro.

\begin{tabular}{|c|c|c|}
\hline 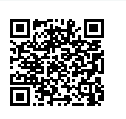 & (c) (i) (-) & 43 \\
\hline
\end{tabular}




\section{Revista de CIENCIAS AMBIENTALES Tropical Journal of Environmental Sciences}

Revista de Ciencias Ambientales (Trop J Environ Sci) e-ISSN: 2215-3896

(Julio-Diciembre, 2019) . Vol 53(2): 25-46 DOI: https://doi.org/10.15359/rca.53-2.2

Open Access: www.revistas.una.ac.cr/ambientales e-mail: revista.ambientales@una.ac.cr Suárez A., Baldioceda A., Durán G., Rojas J., Rojas D., Guillén, A.

\section{Agradecimientos}

Se agradece a los integrantes de las ASADAS quienes participaron en este estudio. Asimismo, al apoyo económico de los proyectos SIA 0035-15 y SIA 0572-16. El primero se denomina Fortalecimiento de las Capacidades de las Asociaciones Administradoras de Acueductos Rurales (ASADAS), mediante la capacitación e implementación de mejores prácticas, técnicas e innovación en la gestión comunitaria del agua; el segundo, Fondos CONARE-Regionalización, Universidad Nacional. Finalmente, agradecemos a la Revista y a las personas revisoras anónimas, por sus oportunos comentarios al manuscrito.

\section{Referencias}

Alfaro, L. (2018, abril). Gestión de ASADAS. Oficina Regional de Acueductos Rurales, Región Chorotega (ORAC). Presentado en Foro Gobernanza del Agua: Derecho Humano al Agua y Saneamiento, Universidad Nacional Campus Liberia. https://doi.org/10.18235/0001031

APHA. (2017). Standard methods for the examination of water and wasterwater (23. ${ }^{\mathrm{a}}$ ed.; L. L. Bridgewater, R. B. Baird, A. D. Eaton, E. W. Rice, American Public Health Association, American Water Works Association y Water Environment Federation, eds.). Washington, D. C.: American Public Health Association. https://doi.org/10.1002/j.1551-8833.2017.tb00020.x

Astorga, Y. (2016). Gestión del recurso hídrico en Costa Rica. Revista Ambientico, 260, 17-24.

AyA. (2015). Política de Organización y Fortalecimiento de la Gestión Comunitaria de los Servicios de Agua Potable y Saneamiento. San José, Costa Rica: Instituto Costarricense de Acueductos y Alcantarillados

AyA. (2016). Política Nacional de Agua Potable de Costa Rica, 2017-2030. San José, Costa Rica: Instituto Costarricense de Acueductos y Alcantarillados. Recuperado de https://www.AyA. go.cr/Noticias/Documents/AyA\%20Pol\%C3\%ADtica\%20Nacional\%20de\%20Agua\%20Potable\%20de\%20Costa\%20Rica\%202017-2030.pdf

Chamizo, H. A. y Mora, D. (2006, diciembre). Estudio ecológico de las enfermedades de transmisión hídrica en la cuenca hidrográfica superficial del río grande de Tárcoles. Revista Costarricense de Salud Pública, 29, 8-24. https://doi.org/10.1590/s1020-49891998000900007

Coto, M. (2016). Paacume: más que un proyecto de infraestructura, un proyecto de desarrollo. Revista Ambientico, 260, 32-37.

Custodio, E. y Llamas, M. R. (1996). Hidrología subterránea (2. ${ }^{a}$ ed. corr.). Barcelona: Ed. Omega.

De Stefano, L. y Llamas, M. R. (Eds.). (2013). Water, agriculture and the environment in Spain: can we square the circle? Boca Ratón: CRC Press.

Grey, D. y Sadoff, C. W. (2007). Sink or Swim? Water security for growth and development. Water Policy, 9(6), 545-571. https://doi.org/10.2166/wp.2007.021

\begin{tabular}{|c|c|c|c|c|c|}
\hline 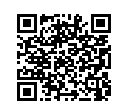 & (c) & $\underset{\text { AMBIENTALIES }}{\infty}$ & $\frac{1 \%}{2 \%}$ & $\frac{\text { UNA }}{\frac{\text { UNIVERSIDAD }}{\text { NACIONAL }}}$ & 44 \\
\hline
\end{tabular}




\section{Revista de CIENCIAS AMBIENTALES Tropical Journal of Environmental Sciences}

Revista de Ciencias Ambientales (Trop J Environ Sci) e-ISSN: $2215-3896$

(Julio-Diciembre, 2019) . Vol 53(2): 25-46 DOI: https://doi.org/10.15359/rca.53-2.2

Open Access: www.revistas.una.ac.cr/ambientales e-mail: revista.ambientales@una.ac.cr Suárez A., Baldioceda A., Durán G., Rojas J., Rojas D., Guillén, A.

GWP. (2011). Situación de los recursos hídricos en Centroamérica: hacia una gestión integrada. GWP Centroamérica, Tegucigalpa. Recuperado de http://www.repo.funde.org/847/

INEC. (2015). Encuesta Nacional de Hogares. Recuperado de http://www.inec.go.cr/wwwisis/ documentos/INEC/ENAHO/ENAHO_2015/ENAHO_2015.pdf

IPCC. (2014). Fresh Water. Climate Change 2014 Impacts, Adaptation, and Vulnerability Part A: Global and Sectoral Aspects Recuperado de https://www.ipcc.ch/site/assets/uploads/2018/02/ WGIIAR5-FrontMatterA_FINAL.pdf

Kuzdas, C. y Wiek, A. (2014). Governance scenarios for addressing water conflicts and climate change impacts. Environmental Science \& Policy, 42, 181-196. https://doi.org/10.1016/j. envsci.2014.06.007

Luna, S., Reyes, L., Chinchilla, M. y Catarinella, G. (2002). Presencia de ooquistes de Cryptosporidium spp. en aguas superficiales en Costa Rica. Parasitol. Latinoam., 57, 63-65. https:// doi.org/10.4067/s0717-77122002000100015

Madrigal-Ballestero, R. y Naranjo, M.A. (2015). Adaptive capacity, drought and the performance of community-based drinking water organizations in Costa Rica. Journal of Water and Climate Change, 6(4), jwc2015154. https://doi.org/10.2166/wcc.2015.154

MIDEPLAN. (2017). Área de Planificación Regional. Costa Rica: estadísticas regionales 2010-2015. Recuperado de http://obturcaribe.ucr.ac.cr/documentos-publicaciones/planes-y-programas/ mideplan-1/312-costa-rica-estadisticas-regionales-2010-2015/file

Montgomery, M. A., Bartram, J. y Elimelech, M. (2009). Increasing Functional Sustainability of Water and Sanitation Supplies in Rural Sub-Saharan Africa. Environmental Engineering Science, 26(5), 1017-1023. https://doi.org/10.1089/ees.2008.0388

Mora, D. y Vargas, C. L. (2013). Informe sobre la presencia de arsénico en algunos acueductos. San José, Costa Rica: Instituto Costarricense de Acueductos y Alcantarillados (AyA).

Moriarty, P., Smits, S., Butterworth, J. y Franceys, R. (2013). Trends in Rural Water Supply: Towards a Service Delivery Approach. Water Alternatives, 6(3), 329-349.

Navarro-Garro, A., Murillo, D. P., Segura, C. M. y Ugalde, M. E. (2013). Vulnerabilidad de los sistemas de acueductos rurales: cómo identificarla. Tecnología en Marcha, 26(3), 62-73. https://doi.org/10.18845/tm.v26i3.1518

OECD. (2013). Water security for better lives. A summary for policymakers. Recuperado de https:// www.oecd.org/env/resources/Water\%20Security\%20for\%20Better\%20Lives-\%20brochure.pdf

OMS. (2013). Progresos en materia de saneamiento y agua potable: informe de actualización. Recuperado de https://apps.who.int/iris/bitstream/handle/10665/204485/9789243509143_spa. pdf;jsessionid=47A333F4C18FB154BDAC2FEDB896D4F6? sequence $=1$

\begin{tabular}{|c|c|c|}
\hline 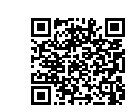 & (c) (i) (5) & 45 \\
\hline
\end{tabular}




\section{Revista de CIENCIAS AMBIENTALES Tropical Journal of Environmental Sciences}

Revista de Ciencias Ambientales (Trop J Environ Sci) e-ISSN: 2215-3896

(Julio-Diciembre, 2019) . Vol 53(2): 25-46 DOI: https://doi.org/10.15359/rca.53-2.2 Open Access: www.revistas.una.ac.cr/ambientales e-mail: revista.ambientales@una.ac.cr Suárez A., Baldioceda A., Durán G., Rojas J., Rojas D., Guillén, A.

Presidencia de la República. (2014, 10 de octubre). Decreto 38642 MP-MAG. Recuperado de http://www.pgrweb.go.cr/scij/Busqueda/Normativa/Normas/nrm_texto_completo. aspx?param $1=$ NRTC\&nValor $1=1 \&$ nValor2=78091\&nValor3=98281\&strTipM=TC

Prüss-Üstün, A., Gore, F., Bartram, J. y Bos, R. (2008). Safer water, better health: costs, benefits and sustainability of interventions to protect and promote health. Recuperado de https://apps. who.int/iris/bitstream/handle/10665/43840/9789241596435_eng.pdf?sequence $=1$

Ramírez, A. (2007). Conflictos socioambientales y recursos hídricos en Guanacaste; una descripción desde el cambio en el estilo de desarrollo (1997-2006). Anuario de Estudios Centroamericanos, 33/34, 359-385. https://doi.org/10.15517/aeca.v1i1.28852

Retana, J. (2012). Eventos hidrometeorológicos extremos lluviosos en Costa Rica desde la perspectiva de la adaptación al cambio en el clima. Revista de Ciencias Ambientales, 44(2), 5-16. https://doi.org/10.15359/rca.44-2.1

Sánchez-Murillo, R., Durán-Quesada, A., Birkel, C., Esquivel-Hernández, G. y Boll, J. (2016). Tropical precipitation anomalies and d-excess evolution during El Niño 2014-16. Hydrological Processes, 31, 956-967. https://doi.org/10.1002/hyp.11088

Sandoval-Moreno, A. y Günther, M. G. (2013). La gestión comunitaria del agua en México y Ecuador: otros acercamientos a la sustentabilidad. Revista de Sociedad, Cultura y Desarrollo Sustentable, 9(2), 165-179.

UNESCO. (2017). Informe de las Naciones Unidas sobre los recursos hídricos en el mundo 2015. [Resumen ejecutivo]. Recuperado de https://unesdoc.unesco.org/ark:/48223/pf0000232272_spa

World Health Organization. (2012). Water safety planning for small community water supplies: step-by-step risk management guidance for drinking-water supplies in small communities. Geneva: World Health Organization. https://doi.org/10.1201/9780203022870.ch8

World Water Forum y Global Water Partnership. (2000). Towards water security: a framework for action. Stockholm: GWP.

Zeitoun, M. (2011). The Global Web of National Water Security: The Global Web of National Water Security. Global Policy, 2(3), 286-296. https://doi.org/10.1111/j.1758-5899.2011.00097.x

Zeledón, J. M. (2016). Agua para Guanacaste: PIAAG. Revista Ambientico, 260, 25-31. Recuperado de www.ambientico.una.ac.cr

\begin{tabular}{|c|c|c|}
\hline 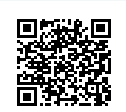 & 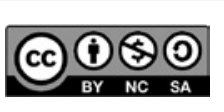 & 46 \\
\hline
\end{tabular}

